\title{
Possibility of "Comet-Like" Transport of Acetylcholine Receptors in Command Helix Neurons in Cellular Analog of Habituation
}

\author{
Arkady S. Pivovarov ${ }^{1}$, Galina B. Murzinaa ${ }^{2}$, Denis A. Makhnovsky ${ }^{1}$, Mariya S. Tret'yakova ${ }^{1}$ \\ ${ }^{1}$ Department of Higher Nervous Activity, Faculty of Biology, Moscow Lomonosov State University, Moscow, \\ Russia \\ ${ }^{2}$ Institute of Higher Nervous Activity and Neurophysiology, Russian Academy of Sciences, Moscow, Russia \\ Email: ${ }^{*}$ as pivovarov@mail.ru
}

Received 22 January 2014; revised 22 February 2014; accepted 2 March 2014

Copyright @ 2014 by authors and Scientific Research Publishing Inc.

This work is licensed under the Creative Commons Attribution International License (CC BY). http://creativecommons.org/licenses/by/4.0/

(c) (i) Open Access

\begin{abstract}
The presence of "comet-like" radial transport of acetylcholine receptors by actin microfilaments without the participation of myosin motors in the depression of acetylcholine-induced inward chloric current (ACh-current) in command neurons of defensive behavior of the land snail, Helix lucorum, in a cellular analog of habituation was investigated. For that purpose the effects of CK548, CK-636 (inhibitors of actin-related protein complex Arp2/3, whose activation triggers rapid actin polymerization and the formation of the "comet-like" tail on the actinic filament) and wiskostatin (an N-WASP protein inhibitor, activating Arp2/3) on the depression of ACh-current were studied. The attenuation of ACh-current depression was observed upon the addition of CK548. At the same time, CK-636 and wiskostatin irreversibly strengthened the depression of this current and suppressed its spontaneous recovery. The results of CK-548 action and its mathematical modeling allow suggesting the presence of "comet-like" transport of acetylcholine receptors, initiated by Arp2/3 protein complex in receptor endo- and exocytosis in command neurons of $\mathrm{He}$ lix lucorum in a cellular analog of habituation. Irreversible inhibition of vital metabolic processes of the neuron by wiskostatin and CK-636, which lead to the decrease in the level of ATP, could have caused irreversible effects of these blockers on current depression.
\end{abstract}

\section{Keywords}

CK-548, CK-636, Wiskostatin, Depression of Acetylcholine-Induced Inward Current, Command Neurons, Helix lucorum, Cellular Analog of Habituation

\footnotetext{
"Corresponding author.
}

How to cite this paper: Pivovarov, A.S., Murzina, G.B., Makhnovsky, D.A. and Tret'yakova, M.S. (2014) Possibility of “Comet-Like" Transport of Acetylcholine Receptors in Command Helix Neurons in Cellular Analog of Habituation. World Journal of Neuroscience, 4, 133-143. http://dx.doi.org/10.4236/wjns.2014.42016 


\section{Introduction}

Habituation is one of the forms of learning, when a repeated stimulus that is constant in value causes a gradual reversible decrease of an animal's response [1]. Habituation teaches animals to ignore stimuli that are no longer new or have lost their biological significance. The repeated tactile stimulation of Helix lucorum leads to a reversible decrease in the level of the animal's defensive response [2]. We are investigating the cellular mechanism of habituation on command neurons' defensive behavior of Helix lucorum by using cellular analog of habituation. Repeated tactile stimulus is replaced by rhythmic application of equal portions of acetylcholine (ACh) to the soma of a command neuron. Instead of the animal's defensive behavior we are recording the command neuron's electric reaction (inward current induced by ACh, ACh-current) [3] caused by the movement of chloric ions through the membrane [4].

According to the research, reversible suppression of ACh-current of Helix lucorum's command neurons' defensive behavior in habituation cellular analog depends on the reduction of membrane acetylcholine receptors (AChR) in the ACh application zone due to the endocytosis intensification and exocytosis attenuation [5] [6]. These changes in the number of membrane receptors take place by involving the cytoskeleton elements-actin microfilaments and microtubules [5] [7] [8], as well as neuronal enzyme systems—protein kinases and protein phosphatases [8]-[10]. Two mechanisms of actin microfilament participation in endo- and exocytosis are possible. The first mechanism ensures receptor transport by motor proteins myosins, traveling along the actin filaments in either direction by means of vesicles. The other proposed mechanism operates without myosin motors through the process of actin polymerization. In this case, extracellular signal is transmitted to actin cytoskeleton via membrane receptors and G-proteins, leading to rapid actin polymerization in the zones of lamellipodium and filopodium formation (various types of pseudopodia) or to rapid polymerization of tension fibers that transect a cell of actin filament bundles. Rapid lengthening of actin filaments is accompanied by the appearance of a driving force in the direction of the filament growth. This type of rapid actin polymerization takes place during the movement of fibroblast cells and of certain lower fungi with the assistance of pseudopodia in the direction of (or opposite to) the significant signal. During polymerization the actin monomers form a helically-coiled polar filament with differing ends: a pointed (minus) end and a barbed (plus) end. The initial polymerization stage ("nucleation") transforms into the faster stage when monomers bind to the plus end and dissociate from the pointed (minus) end. Actin-binding proteins (CAP proteins) can block an actively growing plus end, while the other proteins can cut actin filaments, forming new fragments or depolymerize the actin [11] [12].

A cell has several actin-related proteins (Arp)—Arp1, Arp2, Arp3. It has been discovered that Arp1 is involved in the movement of organelles along microtubules, while Arp2 and Arp3 make up the Arp2/3-complex, which has an increased affinity to actin filament minus ends. This complex can both serve as a matrix, where the new actin filaments formation (nucleation) takes place, and bind to the lateral sides of the filaments, ensuring their branching. The rapidly growing plus ends of actin filaments are generally turned toward cell periphery, pushing the membrane forward. Arp2/3-complex speeds up actin polymerization by 2 - 3 times. Other proteins are required to activate this complex. The Wiskott-Aldrich Syndrome Protein (WASP) is one of such activating proteins. WASP is the effector of Rho GTPase Cdc42 and a key component of signal pathways that regulate actin cytoskeleton [11] [13] [14]. Monomer actin and Arp2/3-complex binding domains are located next to each other at the C-end of the WASP-protein. This ensures their spatial vicinity and can facilitate the process of actin polymerization. G-proteins and other signaling molecules binding domains are located at the N-end of the WASP-protein. This guarantees WASP and its analogs the ability to serve as intermediaries under the influence of numerous forces and the involvement in the transmission of extracellular signal (on the segment between G-proteins and Arp2/3-complex), which activates actin polymerization. The "comet-like tail" (CT) is created from the continuously polymerizing actin, whose formation is regarded to ensure intracellular vesicle transport (endocytosis) without the participation of motor proteins [11] [12] [14].

If motor protein sustained transport processes are conducted along the dynamically stable actin filaments (when the proceeding actin polymerization-depolymerization processes are at an equilibrium), then in the case of "comet-like" movement, additional activation of ATP-G-actin monomers is required to initiate a force that would affect the object in motion. So far, there hasn't a single mathematical model out there that can fully describe how chemical energy within a cell, which is released in the process of actin polymerization, and it is transformed into the energy of force that pushes the moving surface. [15]. The ATP hydrolysis role in the mechanism of actin filament branching also has not yet been sufficiently studied. Different molecular models of 
cellular organelle motility are based on the opposite assumptions whether the interaction of individual filaments with the filament end is an autocatalytic process or not. According to the models, this difference leads to different force-speed ratios: low speed even with great force in an autocatalytic model and rapidly gained speed even with little force in a "nucleating" model [16].

As shown by the studies, "comet-like” movement is characteristic for pathogenic bacteria Licteria monocytogenes, Shingella flexneri and cowpox virus [17] [18], which are instead of WASP covered by different protein ActA, performing the same function. Both proteins promote the interaction of G-actin and Arp2/3-complex. This interaction leads to the formation and branching of actin filaments. The formation of "comet-like tail" has been observed in Xenopus oocytes; furthermore, WASP was found on the surface of every vesicle connected to CT. Morphologically these vesicles are identical to cell endosomes [19]. Significant reduction in endosome motility is observed in HeLa cells. RBL cells demonstrate even a complete blockage of pinosomes actin-dependent motility during the mutation of Ca-binding protein annexin 2 [20], which is capable to bind with phosphatidylinositol 4,5-biphosphate (which is part of the mobile vesicle membrane) and F-actin [21]. In neurons "comet-like" movement can play a role in cell restructuring e.g. in the cytoplasm adjacent to the section of the membrane that received the extracellular "signal". In this case it will result in a launch of an entire cascade of membrane and intracellular processes (one of which is additional activation of actin monomers) and finally lead to the change of motility and structure of that section of the cell e.g. growth of filopodia or the formation of new spinelets [22].

Our earlier results allowed us to suspect an existence of radial acetylcholine receptor transport with the involvement of myosin motors along actin filaments in command neurons of a land snail (Helix lucorum) in a cellular analog of habituation [23]. Given that the presence of N-WASP protein was shown in the neurons of marine mollusk Aplysia [24], the possibility of the second aforesaid mechanism of receptor transport by actin filaments (without myosin involvement) in the neurons of a land mollusk cannot be excluded. This time we focused on testing empirically the possible role of "comet-like" acetylcholine receptor transport, activated by WASP and Arp2/3 proteins in the molecular mechanism of change in inward chlorine ACh-current of command neurons of land snail's defensive behavior in a cellular analog of habituation. The research was performed by means of inhibitory analysis in electrophysiological experiments as well as mathematical modeling.

\section{Materials and Methods}

\subsection{Animals}

Snails, Helix lucorum, were collected locally in the Sevastopol region, the Crimea, Ukraine. Experiments were performed using isolated ganglia preparation at room temperature $\left(18^{\circ} \mathrm{C}-22^{\circ} \mathrm{C}\right)$.

Animals were anaesthetized for $30 \mathrm{~min}$ in cold saline containing ice and then the circumoesophageal nerve ring was removed for study. Circumoesophageal ganglia were pinned down by steel microneedles, dorsal side up, on a Sylgard (Dow-Corning) coated flow chamber, with a bath volume of $1 \mathrm{ml}$. Chamber was filled with normal Helix saline (mM): NaCl-100, KCl-4; $\mathrm{CaCl}_{2}-10$; $\mathrm{MgCl}_{2}-4$, Tris buffer, 10; adjusted to $\mathrm{pH} 7.5$ - 7.7 with $\mathrm{HCl}$. After treatment of the peripharyngeal nerve ring of ganglia with enzyme (digestase/Seatec/, Russia-Luxembourg, $8 \mathrm{mg} / 1.2 \mathrm{ml}, 20-70 \mathrm{~min}$ at room temperature: $18^{\circ} \mathrm{C}-22^{\circ} \mathrm{C}$ ) connective tissue sheath covering the ganglia was removed.

\subsection{Registration of Transmembrane Ionic Currents}

Experiments were carried out on identified LPa2, LPa3, RPa3 and RPa2 neurons from the left and right parietal ganglia of Helix lucorum. These cells are command elements of withdrawal responses to noxious stimuli [25]. Cell membrane contains extrasynaptic [26] and postsynaptic [27] [28] cholinergic receptors.

Single-barrel glass microelectrodes were pulled using a PUL-1 puller (World Precision Instruments, USA) from Pyrex glass (1.5 mm outer diameter) and filled with $2 \mathrm{M}$ potassium acetate; resistance 4 - $44 \mathrm{M} \Omega$ (19.48 \pm $1.02 \mathrm{M} \Omega$; mean \pm S.E.M.). The electrodes were connected by an Ag-AgCl microelectrode holder (World Precision Instruments design) to a Micro-Electrode Amplifier MEZ-8101 (Nihon Kohden) and Voltage Clamp Amplifier CEZ-1100 (Nihon Kohden) that were used in Virtual Ground Mode for two-electrode voltage clamp experiments. A briquette of Ag-AgCl (Medicor) was used as a reference electrode. The neurons were clamped at $-75 \mathrm{mV}$. Currents were entered on a PC through the analogous-digital interface L-154 (L-CARD, Moscow) and 
recorded using CONAN 3.5 software (InCo, Moscow, Russia). In the experiment currents were also recorded on a KSP-4 ink recorder to assess their dynamics in real time.

\subsection{Test Stimulation}

ACh was applied ionophoretically (interstimulus interval $5 \mathrm{~min}$ ) onto the neuronal soma using the output of the Laboratory Electrostimulator ESL-2 (Kaunas Research Institute of Radiometrical Engineering) isolated from the ground. The ionophoretic solution was as follows: $1 \mathrm{M}$ ACh chloride (Sigma) in distilled water (pH 7.0). The resistance of the ionophoretic pipette was 10 - $45 \mathrm{M} \Omega$. The reference pipette was filled with normal Helix saline with a resistance of 1 - $5 \mathrm{M} \Omega$. Cationic currents (200 - $500 \mathrm{nA}$; 0.3 - $3.0 \mathrm{~s}$ ) were passed through a pipette containing a solution of ACh chloride from the device connected to Electrostimulator ECL-2. Inward current (AChcurrent) with amplitude up to $50 \mathrm{nA}$ was recorded in response to ACh iontophoresis (Figure 1(A)). Ejection of positive currents through an ionophoretic pipette filled only with distilled water had no effect on the cells. A negative retention current (10 nA) was passed continuously through the ionophoretic pipette in order to prevent spontaneous diffusion of ACh.

To estimate the magnitude of input resistance of the neuron the amplitude of the leakage current caused by hyperpolarizing shift $(10 \mathrm{mV})$ of the holding potential was measured. Rectangular current pulse duration of $5 \mathrm{~s}$ from the output of the electrical stimulator ECL-2 for $10 \mathrm{~s}$ prior to application of ACh (Figure 1(A)) was used. Input resistance of neurons was $2-8 \mathrm{M} \Omega$.

\subsection{Drugs}

CK-548, CK-636, inhibitors of the Arp2/3 protein complex, which activates the formation of "comet-like tail" on the ends of actin microfilaments, and wiskostatin, an inhibitor of N-WASP protein, which activates the Arp2/3 protein complex, were used in this paper. All compounds were of Sigma production.

Substances were diluted in dimethyl sulphoxide (DMSO, Sigma) and applied extracellularly. Maximum expected concentration of DMSO in the flow chamber was $0.1 \%$. Substance exposure prior to the start of the series was $60 \mathrm{~min}$. All series were conducted in stopped-flow conditions.

\subsection{Experimental Series}

The series were begun following stabilization of ACh-current amplitude in the course of background testing-
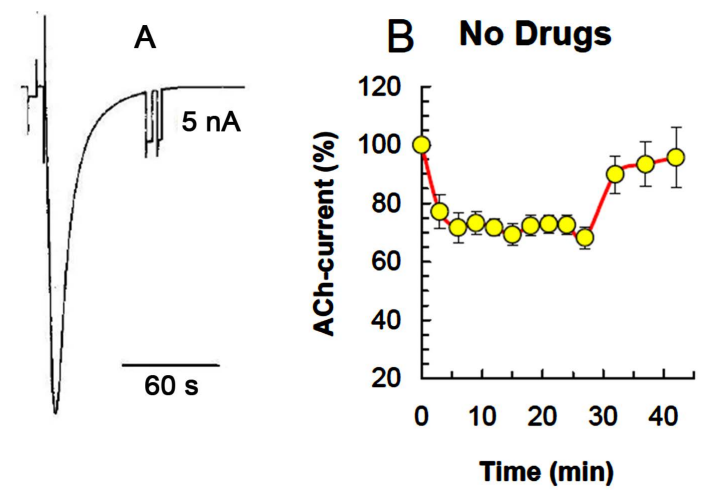

Figure 1. The dynamics of neuron ACh-current depression in a cellular analog of habituation. A: example of inward current evoked by ionophoretic application of acetylcholine to LPa2 neuron. Holding potential $(-75) \mathrm{mV}$. B: ACh-current depression curve without pharmacological effect, constructed basing on the results of all the experiments. Horizontal axis-time, min; Vertical axis-ACh-current amplitude (mean \pm S.E.M.) in \% relative to the ACh-current value in response to the first ACh application in the series, vertical lines-standard error of the mean. 
periodically delivering ACh to neuron soma with a 5 min interval. Stimulation protocol in cellular analog of habituation included 13 applications of equal portions of ACh. The first 10 stimuli of the series were applied with 3-min intervals, while stimuli 11 - 13 were applied with 5-min intervals. Degree and rate of the spontaneous recovery in a series of reduced ACh-current were assessed based on the values of ACh-current at ACh applications 11 - 13. The kinetics of changes in ACh-current in the control series (without pharmacological effects) included the following phases: 1) the current decay phase; 2) the plateau phase of current amplitude stabilization at a reduced level, and 3) the spontaneous current recovery phase (Figure 1(B)).

The results were obtained for 49 neurons ( $4 \mathrm{LPa} 2,16 \mathrm{LPa} 3,22 \mathrm{RPa} 3,7 \mathrm{RPa} 2)$ in 49 experiments. The membrane potential of cells was $(-75)-(-22) \mathrm{mV}(-56.06-1.12 \mathrm{mV})$; the holding potential was $(-75) \mathrm{mV}$.

\subsection{Statistical Analyses}

Normalized values are presented as an arithmetic mean of the sample \pm standard error of the mean. Computer programs Systat 13, Sigma Stat 3.5 (Systat Software, Inc., USA) were used for statistical processing. The significance of group differences was evaluated beforehand by using one way repeated measures analysis of variance (One Way RM ANOVA). If authentic effect differences were found in groups, pairwise comparisons of group means were conducted (blocker-assisted and against control groups) by using Fisher's LSD test. Nonparametric Mann-Whitney Rank Sum test was used to compare average ACh-current amplitudes in two groups (experimental and control) after equal time delays following the start of the series. Differences were considered statistically significant with $P \leq 0.05$.

\subsection{Mathematical Modeling}

A mathematical model that takes into account different receptor locations-on cell membrane and extracellular, inside the formed and recycled vesicles—-was used to analyze experimental data. Also taken into account were the transport of receptors from endoplasmic reticulum to the membrane and the process of receptor degradation. Transfer of receptors between different states were determined by the rate of membrane receptor transport to forming vesicles $\left(k_{1}\right)$ and the rate of their possible yield from those vesicles $\left(k_{2}\right)$, rates of endocytosis $\left(k_{3}\right)$ and exocytosis $\left(k_{4}\right)$ receptors, rate of receptor transport from endoplasmic reticulum $\left(k_{6}\right)$, and the rate of receptor degradation $\left(k_{5}\right)$. The choice of rate constants of transition was based on experimental data, according to which the time constant of receptor endocytosis was about several minutes, while that of exocytosis was $2-3$ times longer. Mathematical modeling came down to solving a differential equation system that described the change of receptor location. The equation parameters were the rates of change in receptor location. Based on the degree of parameter variation, we made conclusions about the influence of investigated inhibitors on various transfer of receptor.

\section{Results}

\subsection{Effects of CK-548, CK-636 and Wiskostatin on ACh-Current in a Cellular Analog of Habituation}

The effects of blockers of the dynamics of ACh-current amplitude during a series of repeat ACh applications were analyzed: 1) average rate of ACh-current decay it approached the plateau phase; 2) level (degree) of AChcurrent depression (current amplitude in the plateau phase); 3) degree of spontaneous recovery of ACh-current amplitude.

Group differences of substance effects on the level of ACh-current depression and its spontaneous recovery (following the cessation of a series of frequent ACh applications, in comparison with background) were highly significant (one-way RM ANOVA, $F[4,34]=186.728, P<0.001 ; F[4,32]=4.431, P=0.009$ ). ANOVA did not reveal significant group effect of the used inhibitors on the rate of ACh-current depression $(F[4,44]=1.645, P=$ 0.189). The results of a pairwise group comparison (post blocker effect and in the control group) are presented in the Table 1.

CK-548 ( $\mathrm{n}=9,50 \mu \mathrm{M})$ reduced the rate (nonsignificant) and decreased the degree of ACh-current depression $(\mathrm{P}<0.001)$ without changing spontaneous current recovery (Table 1, Figure 2).

CK-636 $(\mathrm{n}=10,50 \mu \mathrm{M})$ and wiskostatin $(\mathrm{n}=8,5 \mu \mathrm{M})$ had a similar effects on the ACh-current depression curve-they increased the degree of depression $(P<0.001, P<0.001)$, while decreasing its level, but did not 


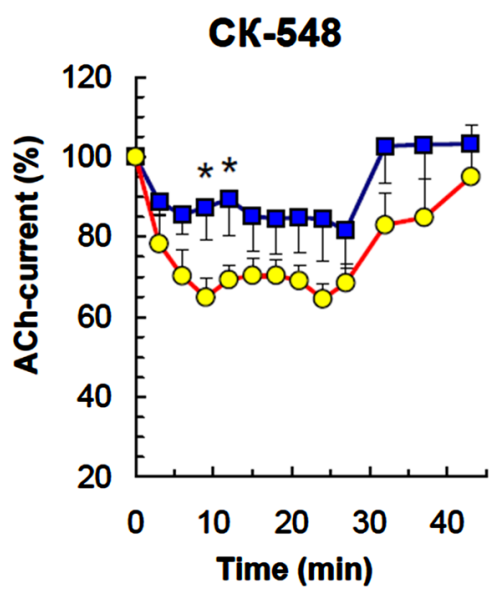

Figure 2. The effect of CK-548, Arp2/3 protein complex inhibitor, on the $\mathrm{dy}$ namics of neuron ACh-current depression in a cellular analog of habituation. The Figure presents overall results from all experiments. Horizontal axis-time, min; vertical axis-ACh-current amplitude (mean \pm S.E.M.) in \% relative to the ACh-current value in response to the first ACh application in the series. Squares-CK-548 $(50 \mu \mathrm{M})$, circles is DMSO (0.1\%, control).Vertical linesstandard error of the mean, ${ }^{*} P<0.05$ - significance level for ACh current amplitude differences between experimental and control groups (Mann-Whitney criterion).

Table 1. Effect of inhibitors of "comet-like” receptor transport on ACh-current depression in a cellular analog of habituation.

\begin{tabular}{|c|c|c|c|c|}
\hline \multirow{2}{*}{$\mathrm{N}$} & \multirow{2}{*}{ Inhibitor } & \multicolumn{2}{|c|}{ ACh-current depression } & \multirow{2}{*}{$\begin{array}{c}\text { ACh-current } \\
\text { strength } 15 \mathrm{~min} \\
\text { after the series, \% }\end{array}$} \\
\hline & & Rate of depression, \%/min & Level of depression, \% & \\
\hline 1 & No drugs; $n=11$ & $4.55 \pm 0.87$ & $71.21 \pm 0.62$ & $95.58 \pm 10.28$ \\
\hline 2 & DMSO, $0.1 \%$, out; (Control); $\mathrm{n}=10$ & $4.97 \pm 0.87$ & $68.38 \pm 1.92$ & $95.68 \pm 11.32$ \\
\hline 3 & CK-548, $50 \mu \mathrm{M}$ + DMSO, $0.1 \%$, out; $\mathrm{n}=9$ & $2.41 \pm 0.79$ & $85.34 \pm 3.09^{* * *} \uparrow$ & $103.2 \pm 8.12$ \\
\hline 4 & CK-636, $50 \mu \mathrm{M}$ + DMSO, $0.1 \%$, out; $\mathrm{n}=10$ & $4.53 \pm 0.80$ & $43.93 \pm 4.73^{* * *} \downarrow$ & $38.41 \pm 8.87^{*} \downarrow$ \\
\hline 5 & Wiskostatin, $5 \mu \mathrm{M}$ + DMSO, $0.1 \%$, out; $\mathrm{n}=8$ & $3.85 \pm 2.13$ & $54.18 \pm 6.08^{* * *} \downarrow$ & $36.63 \pm 21.15^{*} \downarrow$ \\
\hline
\end{tabular}

Note. The Table presents normalized mean values \pm S.E.M.; $n-$ number of neurons in a series; out-extracellular administration of inhibitors. ${ }^{*} P<$ $0.05 ;{ }^{* * *} P<0.001$ (Fisher's LSD test) with pairwise comparison of mean values in experiment and control groups, which were conducted in the event that reliable group change of current depression parameter was obtained based on the results of a One Way RM ANOVA. Arrows indicate the direction of parameter change.

change the rate of depression. Both blockers suppressed spontaneous recovery of ACh-current amplitude $(P=$ 0.039, $P=0.019$ ) (Table 1, Figure 3 and Figure 4). Moreover, in a number of experiments wiskostatin repressed ACh-current completely until the end of the series with no recovery. Irreversible suppression of AChcurrent in series with CK-636 and wiskostatin could be the result of blocker concentrations used in the experiment, where they caused a cytotoxic side effect. To exclude that possibility, we checked the effects of these compounds in lower concentrations: CK-636 $(25 \mu \mathrm{M})$, wiskostatin $(2.5 \mu \mathrm{M}, 1.0 \mu \mathrm{M}, 0.5 \mu \mathrm{M})$. Lowering CK-636 and wiskostatin concentrations did not cancel the irreversible ACh-current depression in a series. 


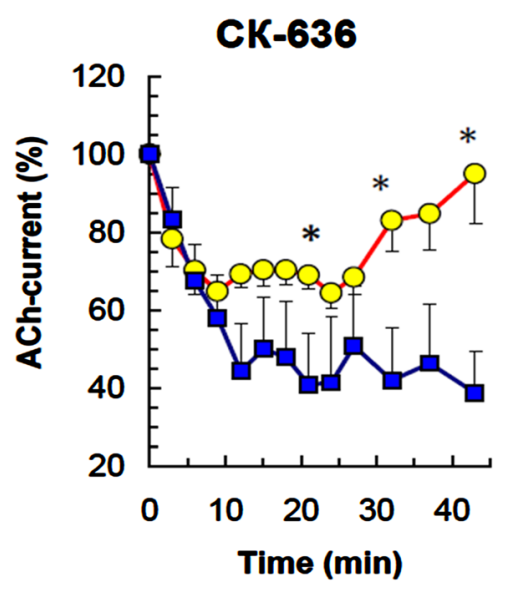

Figure 3. The effect of CK-636, Arp2/3 protein complex inhibitor, on the dynamics of neuron ACh-current depression in a cellular analog of habituation. The Figure presents overall results from all experiments. Horizontal axis-time, min; vertical axis-ACh-current amplitude (mean \pm S.E.M.) in \% relative to the ACh-current value in response to the first ACh application in the series. Squares - CK-636 $(50 \mu \mathrm{M})$, circlesDMSO ( $0.1 \%$, control). Other notations are the same as in Figure 2.

\section{Wiskostatin}

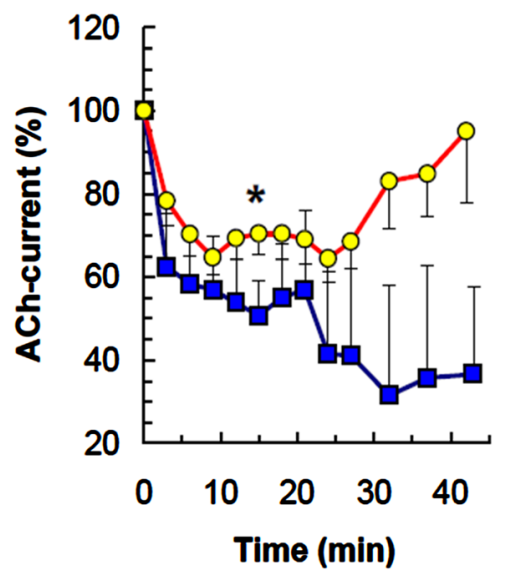

Figure 4. The effect of wiskostatin, NWASP protein inhibitor, on the dynamics of neuron ACh-current depression in a cellular analog of habituation. S quares - wiskostatin (5 $\mu \mathrm{M})$, circles-DMSO $(0.1 \%$, control). Other notations are the same as in Figure 2.

\subsection{Calculated Curves of the Action of CK-548, СK-636 and Wiskostatin on the Number of Membrane Acetylcholine Receptors in a Cellular Analog of Habituation}

Results for the effect of CK-548, obtained by a mathematical model, which we used earlier for modeling the 
impact of various transport process blockers on ACh-current depression, showed that it is possible to obtain a theoretical curve similar to the experimental curve by decreasing the rate of location changes for receptors $\mathrm{k}_{3}, \mathrm{k}_{6}$ by 1.1 , and $\mathrm{k}_{1}, \mathrm{k}_{4}$ by 1.5 and 1.3, respectively (Figure 5, curve 2). For the effect of CK-636 and wiskostatin the theoretical curves similar to the experimental ones were obtained with a significantly smaller reduction of all rates of change (Figure 5, curves 3,4). To obtain curve 3 (which modeled the effect of CK-636) rate constants were solely reduced by 4 - 6 times, while to obtain curve 4 (which modeled the effect of wiskostatin) we applied two-stage reduction of rate constants-an initial 1.5-fold reduction and a secondary 4 - 6-fold reduction 20 minutes later (as for curve 3) (Figure 5).

\section{Discussion}

In planning this experiment, we suggested that the use of inhibitors of key proteins of "comet-like” movementi.e., wiskostatin $\left(\mathrm{IC}_{50}=5 \mu \mathrm{M}\right.$ [29] [30]), described as a selective and reversible N-WASP protein inhibitor, as well as CK-548 and CK-636-inhibitors of the actin-related protein complex Arp2/3, will help us to determine the role of an alternative method of endo- and exocytosis of receptor-containing vesicles). According to research, the actin polymerization rate (dependent on Arp2/3 protein complex activity) declined respectively by 2.3 and 1.6 times after CK-548 and CK-636 at $50 \mu \mathrm{M}$ treatment [31]. CK-636 is inbuilt between Arp2 and Arp3, inhibiting the formation of their active complex, while CK-548 binds itself to Arp3 on its hydrophobic nucleus, which leads to the change of conformation, which blocks the appearance of branched polymerization of actin and "comet-like" transport.

However, the analysis of the obtained results showed that whereas the uses of CK-548 allows us to examine the possibility of the alternative method of vesicle movement, the use of wiskostatin and CK-636 raises the issue of nonselectivity of these inhibitors. Thus, according to mathematical modeling, the reduction of the rate of ACh-current depression stabilization caused by CK-548 is associated with a $1.1-1.5$-fold reduction of receptor transport rates. Since the reduction applies not only to receptor endocytosis, but also to their exocytosis and transport along the membrane to forming vesicles, and it raises the question about the mechanisms that lie at the basis of such a reduction. We know that the binding of Arp2/3 complex to the lateral sides of actin filaments leads to their branching [11]. That is why a reduction of such branching (as a result of inhibitor effect) can lead to a reduction of both the rate of receptor transport with the use of motor proteins and the rate of "comet-like" movement. The results obtained allow us to suggest the possibility that "comet-like" radial transport of extrasynaptic acetylcholine receptors in Helix neurons is involved in a cellular analog of habituation. However, in this study, it is not possible to draw a conclusion about the share of each of the two types of vesicle-containing receptors. We can't count it because 1) rates of receptor transport along the membrane and of receptor exocytosis change along with receptor endocytosis rate change; and 2) according to our earlier studies, inhibition of light

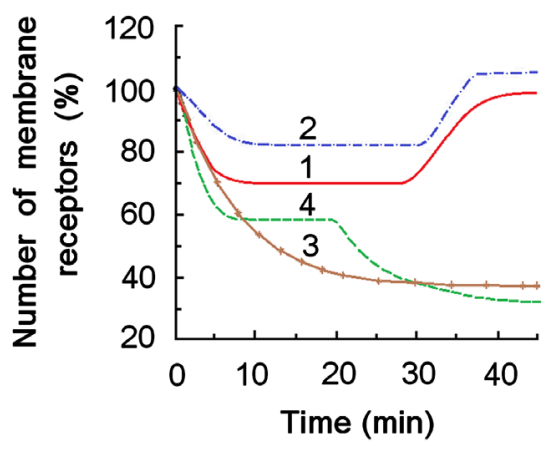

Figure 5. Mathematically modeled curves for the number of membrane acetylcholine receptors which are assumed to determine the depression of acetylcholine-induced inward current. 1-control (without pharmacological influence and after actions of $0.1 \%$ DMSO, both curves are identical); 2 , 3, 4-after actions of inhibitors CK-548 (2), CK-636 (3), wiscostatin (4). 
myosin chains likewise led to the reduction of the rate of ACh-current depression stabilization [23].

The use of another Arp2/3 complex inhibitor-CK-636 and a N-WASP protein blocker, wiskostatin, in our work had another effect-an irreversible suppression of ACh-current until its complete blockage in individual experiments. Decrease in CK-636 and wiskostatin concentrations did not change their effects. Mathematical modeling showed that the correspondence between the theoretical and experimental curves can be obtained by reducing all rates of receptor transfer between various cell parts by 4 - 6 times. Such a significant reduction of all transfer rate constants simultaneously allows us to speak about these inhibitors blocking the processes vital to the neuron, which affect substance transfer (as the rate of receptor transfer along the membrane changes as well). The absence of ACh-current recovery following cessation of a frequent ACh application in a series allows us to conclude that this blocking is irreversible.

The hypothesis about nonselective N-WASP blocking by wiskostatin is confirmed by the results of a number of articles. In particular, significant (by 80\%) suppression of induced chloride currents of HT29-CL19A line epithelial cells with wiskostatin in concentrations (5-10 $\mu \mathrm{M})$ [32] is similar to the ones used in our paper. The authors believe this effect to be the result of wiskostatin destroying actin cytoskeleton. The use of wiskostatin in liver cells caused rapid dose-dependent reduction in the level of ATP [33]. In particular, reduction of ATP level by $20 \%$ of control was obtained after a one hour of wiskostatin treatment at $10 \mu \mathrm{M}$ (similar to the wiscostatin concentration in our experiments). The conclusions drawn in the present paper exclude its use as selective $\mathrm{N}$ WASP blocker, since wiskostatin suppresses all liver cell functions effectively and nonselectively, including transmembrane transport, as a result of ATP level reduction. We believe that the concordance of wiskostatin and CK-636 effects on ACh-current depression in our experiments can likewise be due to the reduction of neuron ATP level by these blockers.

It is well known that certain chemical substances are respiration and phosphorylation uncouplers and they lead to a decrease in ATP level inside a cell [34]. Uncouplers are usually lipophilic substances that pass easily through a membrane's lipid layer. One of such substances is 2,4-dinitrophenol. By their stereochemical formulae wiskostatin and CK-636 like 2,4-dinitrophenol are the derivatives of aromatic hydrocarbons, which are capable of protonating. Consequently, they can also be respiration and phosphorylation uncouplers in mitochondria, and lead to the reduction in neuron ATP level.

The different effects of CK-548 and CK-636 on ACh-current depression curve were unexpected to us, since, according to scientific publications, both compounds selectively block the same Arp2/3 complex, even though they belong to two different low molecular compound classes that bind to different sites of Arp2/3, thus preventing its function [31]. It is possible that the small differences in the structure of these inhibitors account for the fact that CK-636 can lead to irreversible decrease in ATP level in neurons, while CK-548 cannot. The comparison of crystal structure of Arp2/3 complex with bound CK-548 and CK-636 [31] proves this assumption. During the interaction with Arp2/3 complex the CK-636 benzene rings have nearly flat structure as well as the CK-548 benzene rings are situated in different planes. Such geometry of benzene rings in CK-548 molecule seems to prevent proton release and therefore CK-548 is not a respiration and phosphorylation uncoupler.

It is possible that a similar irreversible decrease in the ATP level caused by the use wiskostatin is the reason for its non-selectivity, although according to the publications, it is considered extremely selective inhibitor of protein N-WASP. Taking into account this characteristic of wikostatin, we have chosen it as a selective N-WASP blocker for our experiments.

\section{Acknowledgements}

This study was supported by the Russian Foundation for Basic Research (grant 12-04-00209-a).

\section{References}

[1] Rankin, C.H., Abrams, T., Barry, R.J., Bhatnagar, S., Clayton, D.F., Colombo, J., Coppola, G., Geyer, M.A., Glanzman, D.L., Marsland, S., McSweeney, F.K., Wilson, D.A., Wu, C.-F. and Thompson, R.F. (2009) Habituation Revisited: An Updated and Revised Description of the Behavioral Characteristics of Habituation. Neurobiology of Learning and Memory, 92, 135-138. http://dx.doi.org/10.1016/j.nlm.2008.09.012

[2] Nistratova, V.L. and Pivovarov, A.S. (2005) Inositol Triphosphate and Ryanodine Receptors in the Control of the Cholinosensitivity of Common Snail Neurons by the Na,K Pump during Habituation. Neuroscience and Behavioral Physiology, 35, 699-708. http://dx.doi.org/10.1007/s11055-005-0113-2 
[3] Pivovarov, A.S. (2001) Participation of Second Messengers in Decrease of Cholinosensitivity of Command Helix Neurones in Cellular Model of Habituation: Modulatory Effects of Opioids. In: Ayrapetyan, S.N. and North, A.C.T., Eds., Modern Problems of Cellular and Molecular Biophysics, Noyan Tapan, Yerevan, 69-81.

[4] Pivovarov, A.S. and Walker, R.J. (1996) Direct and Modulatory Effects of FMRFamide, SKPYMRFamideand acetylSKPYMRFamide on LPa2, LPa3 and RPa3 Identified Neurons of Helix lucorum. Regulatory Peptides, 67, 169-178. http://dx.doi.org/10.1016/S0167-0115(96)00129-2

[5] Makhnovskii, D.A., Tretyakova, M.S., Pivovarov, A.S. and Murzina, G.B. (2011) Endocytosis of Cholinoreceptors in the Mechanism of the Depression of the Cholinosensitivity of Neurons in the Common Snail in a Cellular Model of Habituation. Neuroscience and Behavioral Physiology, 41, 617-625. http://dx.doi.org/10.1007/s11055-011-9464-z

[6] Makhnovskiy, D.A., Murzina, G.B. and Pivovarov, A.S. (2012) Cholinergic Receptor Exocytosis under Conditions of Depression of Acetylcholine-Induced Current in Edible Snail Neurons in Cellular Analogue of Habituation. Bulletin of Experimental Biology and Medicine, 153, 424-427. http://dx.doi.org/10.1007/s10517-012-1731-7

[7] Pivovarov, A.S., Vasil’yeva, N.A., Murzina, G.B. and Makhnovskii, D.A. (2013) The Role of Actin Microfilaments in Depression of Acetylcholine-Induced Currents in Common Snail Neurons in a Cellular Analog of Habituation. Neuroscience and Behavioral Physiology, 43, 557-564. http://dx.doi.org/10.1007/s11055-013-9771-7

[8] Pivovarov, A.S., Murzina, G.B., Makhnovsky, D.A., Tret’yakova, M.S. and Vasil'yeva, N.A. (2013) Mobility of Acetylcholine Receptors in Command Helix lucorum Neurons in a Cellular Analog of Habituation. Invertebrate Neuroscience, 13, 135-150. http://dx.doi.org/10.1007/s10158-013-0155-z

[9] Makhnovskii, D.A., Murzina, G.B., Tretyakova, M.S. and Pivovarov, A.S. (2013) The Role of Serine/Threonine and Tyrosine Protein Kinases in Depression of the Cholinosensitivity of Neurons in the Common Snail in a Cellular Analog of Habituation. Neuroscience and Behavioral Physiology, 43, 22-33. http://dx.doi.org/10.1007/s11055-012-9686-8

[10] Pivovarov, A.S., Murzina, G.B., Tret'iakova, M.S. and Makhnovskiŭ, D.A. (2013) Role of Serine/Threonine and Tyrosine Protein Phosphatases in Command Helix lucorum Neurons at the Cellular Correlate of Habituation. Zhurnal Vysshei Nervnoi Deiatelnosti Imeni I.P.Pavlova, 63, 256-268.

[11] Klyachko, N.L. (2000) Biological Motility and Actin Polymerization. Sorosovsky Obrazovatelny zhurnal, 6, 5-9.

[12] Apodaca, G. (2001) Endocytic Traffic in Polarized Epithelial Cells: Role of the Actin and Microtubule Cytoskeleton, Traffic, 2, 149-159. http://dx.doi.org/10.1034/j.1600-0854.2001.020301.x

[13] Leung, D.W., Morgan D.M. and Rosen M.K. (2006) Biochemical Properties and Inhibitors of (N-)WASP. Methods in Enzymology, 406, 281-296. http://dx.doi.org/10.1016/S0076-6879(06)06021-6

[14] Takenawa, T. and Suetsugu, S. (2007) The WASP-WAVE Protein Network: Connecting the Membrane to the Cytoskeleton. Nature Reviews Molecular Cell Biology, 8, 37-48. http://dx.doi.org/10.1038/nrm2069

[15] Dickinson, R.B. (2009) Models for Actin Polymerization Motors. Journal of Mathematical Biology, 58, 81-103. http://dx.doi.org/10.1007/s00285-008-0200-4

[16] Dickinson, R.B. and Purich, D.L. (2002) Clamped-Filament Model for Actinbased Motors. Biophysical Journal, 82, 605-617. http://dx.doi.org/10.1016/S0006-3495(02)75425-8

[17] Calier, M.-F., Le Clainche, C., Wiesner, S. and Pantaloni, D. (2003) Actin-Based Motility: From Molecules to Movement. BioEssays, 25, 336-345. http://dx.doi.org/10.1002/bies.10257

[18] Calier, M.-F., Wiesner, S. and Pantaloni, D. (2002) Production of Force and Movement by Polymerization of Actin: Mechanisms and Reconstitution in Vitro. Journal of Biological Physics, 28, 327-333. http://dx.doi.org/10.1023/A:1020353402385

[19] Taunton, J., Rowning, B.A., Coughlin, M.L., Wu, M., Moon, R.T, Mitchison, T.J. and Larabell, C.A. (2000) ActinDependent Propulsion of Endosomes and Lysosomes by Recruitment on N-WASP. The Journal of Cell Biology, 1489, 519-530. http://dx.doi.org/10.1083/jcb.148.3.519

[20] Merrifield, C.J., Rescher, U., Almers, W., Proust, J., Gerke, V., Sechi, A.S. and Moss, S.E. (2001) Annexin 2 Has an Essential Role in Actin-Based Macropinocytic Rocketing. Current Biology, 11, 1136-1141. http://dx.doi.org/10.1016/S0960-9822(01)00321-9

[21] Hayes, M.J., Merrifild, C.J., Shao, D., Ayala-Sanmartin, J., D’Souza Schorey, C., Levine, T.P., Proust, J., Curran, J., Bailly, M., Stephen, E. and Moss, S.E. (2004) Annexin 2 Binding to Phosphatidylinositol 4,5-Biphosphate on Endocytic Vesicles Is Regulated by the Stress Response Pathway. The Journal of Biological Chemistry, 279, 14157-14164. http://dx.doi.org/10.1074/jbc.M313025200

[22] Tada, T. and Sheng, M. (2006) Molecular Mechanisms of Dendritic Spine Morphogenesis. Current Opinion in Neurobiology, 16, 95-101. http://dx.doi.org/10.1016/j.conb.2005.12.001

[23] Pivovarov, A.S., Murzina, G.B., Makhnovsky, D.A., Vasil’yeva, N.A. and Tret'yakova, M.S. (2013) Role of Myosins in Depression of Sensitivity of Helix Neurons to Acetylcholine in a Cellular Analog of Habituation. Zhurnal Vysshei 
Nervnoi Deiatelnosti Imeni I.P.Pavlova, 63, 470-478.

[24] Udo, H., Jin, I., Kim, J.H., Li, H.L., Youn, T., Hawkins, R.D., Kandel, E.R. and Bailey, C.H. (2005) Serotonin-Induced Regulation of the Actin Network for Learning-Related Synaptic Growth Requires Cdc42, N-WASP, and PAK in Aplysia Sensory Neurons. Neuron, 45, 887-901. http://dx.doi.org/10.1016/j.neuron.2005.01.044

[25] Ierusalimsky, V.N., Zakharov, I.S., Palikhova, T.A. and Balaban P.M. (1994) Nervous System and Neural Maps in Gastropod Helix lucorum L. Neuroscience and Behavioral Physiology, 24, 13-22. http://dx.doi.org/10.1007/BF02355648

[26] Pivovarov, A.S. and Drozdova, E.I. (1992) Identification of Cholinoreceptors on the Soma of Snail Neurons RPa3 and LPa3. Neurophysiology, 24, 61-69. http://dx.doi.org/10.1007/BF01053484

[27] Ter-Markaryan, A.G., Palikhova, T.A. and Sokolov, E.N. (1991) Effect of Atropine and d-Tubocurarine on the Monosynaptic Connections between Identified Neurons in the Central Nervous System of the Edible Snail. Neuroscience and Behavioral Physiology, 21, 37-38. http://dx.doi.org/10.1007/BF01184236

[28] Palikhova, T.A., Abramova, M.S. and Pivovarov, A.S. (2006) Cholinergic Sensory Inputs to Command Neurons in Edible Snail. Bulletin of Experimental Biology and Medicine, 142, 275-278. http://dx.doi.org/10.1007/s10517-006-0345-3

[29] Peterson, J.R., Bickford, L.C., Morgan, D., Kim, A.S., Ouerfelli, O., Kirschner, M.W. and Rosen, M.K. (2004) Chemical Inhibition of N-WASP by Stabilization of a Native Autoinhibited Conformation. Nature Structural \& Molecular Biology, 11, 747-755. http://dx.doi.org/10.1038/nsmb796

[30] Bacon, C., Lakics, V., Machesky, L. and Rumsby, M. (2007) N-WASP Regulates Extension of Filopodia and Processes by Oligodendrocyte Progenitors, Oligodendrocytes, and Schwann Cells-Implications for Axon Ensheathment at Myelination. Glia, 55, 844-858. http://dx.doi.org/10.1002/glia.20505

[31] Nolen, B.J., Tomasevic, N. and Russell, A. (2009) Characterization of Two Classes of Small Molecule Inhibitors of Arp2/3 Complex. Nature, 460, 1031-1034. http://dx.doi.org/10.1038/nature08231

[32] Ganeshan, R., Nowotarski, K., Di, A., Nelson, D.J. and Kirk, K.L. (2007) CFTR Surface Expression and Chloride Currents Are Decreased by Inhibitors of N-WASP and Actin Polymerization. Biochimica et Biophysica Acta, 1773, 192-200. http://dx.doi.org/10.1016/j.bbamcr.2006.09.031

[33] Guerriero, C.J. and Weisz, O.A. (2007) N-WASP Inhibitor Wiskostatin Nonselectively Perturbs Membrane Transport by Decreasing Cellular ATP Levels. American Journal of Physiology-Cell Physiology, 292, C1562-C1566. http://dx.doi.org/10.1152/ajpcell.00426.2006

[34] Lehninger, A. (1985) Principles of Biochemistry. Mir, Moscow. 